\title{
Time Dependent Transverse Correlations in the Ising Model in D Dimensions
}

\author{
João Florencio ${ }^{1}$, Surajit $\mathrm{Sen}^{2}$, and M. Howard Lee $\mathrm{Le}^{3,4 *}$ \\ ${ }^{1}$ Departamento de Física, Universidade Federal de Minas Gerais, \\ 30.123-970 Belo Horizonte, MG, Brazil \\ ${ }^{2}$ Physics Department, State University of New York at Buffalo, New York 14260, USA \\ ${ }^{3}$ Physics Department, University of Georgia, Athens, Georgia 30602, USA \\ ${ }^{4}$ Korea Institute for Advanced Study, Seoul 130-012, Korea
}

Received on 10 August, 2000

\begin{abstract}
The Ising model is known widely for studying equilibrium behavior. We show that the model is also useful for studying nonequilibrium behavior in some special situations. The method of recurrence relations has been applied to obtain the time evolution of a non-commuting spin operator. Also obtained are the structure functions and their time dependent behavior. It is shown that the transverse component of the static susceptibility can be obtained from the dynamic results.
\end{abstract}

\section{Introduction}

As is well known, the Ising model has been traditionally studied for a variety of equilibrium properties. Can it be used to study nonequilibrium properties also? Is the Ising model as useful perhaps for studying nonequilibrium behavior?

Our model consists of spin-1/2 operators, localized at $D$ dimensional lattice sites. The internal energy is limited to pairwise coupling of the $z$-components of the spins which are nns. This is a quantum mechanical version of the standard Ising model.

Since the $z$ components of spins commute with one another, all the spin states of the energy are stationary. Thus they do not evolve in time. This is well known as a basic property of the Ising model.

If a transverse external field (better yet just in the $x$-direction) is turned on momentarily, it will couple with the $x$-components of the spins imparting an external energy to the system. If the field is turned off, it is as if a state of the $x$-components of spins has been "created" carrying an extra energy.

The situation that has been created is much like a dynamical picture that is described by linear response theory [1]. Since there is no longer an external field, the averaging is done with respect to the internal energy only.

What happens now to this state of the $x$ components of the spins? Let us consider the spin at just one lattice point (say at 0 ) since they are all equivalent by translational invariance. Evidently $\left[H, S_{0}^{x}\right] \neq 0$. Hence the state of this spin is nonstationary and must now evolve in time unlike the state of $S_{0}^{z}$.

The time evolution here means that this spin state is attempting to restore itself to a stationary state by giving off the acquired energy to its neighboring spins to which it is coupled by the exchange interactions. It attempts to do so by pushing the neighboring spins into a nonstationary state. Since the neighboring spins are already in a stationary state, they resist accepting this energy and return it to the source at site 0 . This process goes on indefinitely. What happens is a localization of the energy, trapped about site 0 . This is unique to the Ising model. In the $X Y$ model, for example, the energy would become delocalized.

The time dependent spin-spin correlation function will thus be periodic, never decaying. The trajectories (see Sec. II) will be closed. The nonequilibrium behavior will not be ergodic in the sense of the usual meaning of that term. The localization may be described in terms of dynamic modes of different frequencies, much like the normal modes of vibration. The complexity of these modes evidently will increase with the coordination number.

How can one study the time dependent behavior in this quantum Ising model? Clearly we must solve the Heisenberg equation of motion. Since the model is Hermitian, this can be done most simply by the recurrence relations method [2-4].

Ordinarily one requires static properties to obtain dynamic behavior. It is thus of interest to note that

\footnotetext{
${ }^{*}$ Corresponding author. E-mail address: mhlee@uga.edu
} 
for localized problems such as in this Ising model some static properties are implied by dynamic properties as we shall demonstrate. We shall concentrate on the $1 D$ model. The dynamics is basically similar to that in $1 D$.

\section{Recurrence relations method}

In this section we shall briefly review the recurrence relations method for solving the Heisenberg equation of motion if a system is Hermitian. This general method was introduced in the early 1980s and there have been a number of applications made since to study the time evolution in a variety of Hermitian many-particle systems [5-16].

We are interested in obtaining the time dependent behavior of a transverse spin-spin correlation function $<S_{0}^{x}(t) S_{0}^{x}(0)>$, where $S_{i}^{\alpha}$ is the $\alpha$ component ( $\alpha=x, y$, or $z$ ) of the spin- $1 / 2$ operator at the $i^{t h}$ lattice site, the brackets denote the canonical ensemble average with respect to the Ising Hamiltonian $H=$ $-J \sum_{(i j)} S_{i}^{z} S_{j}^{z}$, where $(i j)$ denotes $n n$ pairs in a lattice. A more general time dependent correlation function is the relaxation function $\left(S_{0}^{x}(t), S_{0}^{x}(0)\right)$, where the inner product means the Kubo scalar product. Note that if $t=0$, this inner product defines the $x x$ component of the susceptibility. As noted in Section I, $\left[H, S_{0}^{x}\right] \neq 0$. Hence $S_{0}^{x}(t)=\exp (i H t) S_{0}^{x}(t=0) \exp (-i H t) \neq S_{0}^{x}(t=$ $0)$, where we have set $\hbar=1$. The time evolution of the operator $S_{0}^{x}$ can give a complete picture of the time dependent behavior. Hence we shall concentrate on this quantity rather than the structure functions.

Let us denote by $A$ the dynamical variable at $t=0$, i.e., $S_{0}^{x}(t=0)=A$. Since we are interested in $t \geq 0$ only, we can define $A(t)=0$ if $t<0$. According to the recurrence relations method $A(t)$ at $t \geq 0$ may be regarded as a vector in a realized Hilbert space of $d$ dimensions provided that $H$ is Hermitian (as is for the Ising Hamiltonian). Thus, $A(t)$ may be given an orthogonal expansions in this space as

$$
A(t)=\sum_{k=0}^{d-1} a_{k}(t) f_{k} .
$$

Here $\left\{f_{k}\right\}$ is a complete set of basis vectors which span the realized space. That is,

$$
\left(f_{k}, f_{k^{\prime}}\right)=0 \quad \text { if } \quad k^{\prime} \neq k
$$

where the inner product means the Kubo scalar product [3]. Also $\left\{a_{k}(t)\right\}$ is a complete set of linearly independent functions of time. They represent the magnitudes of the projection of $A(t)$ on to the basis vectors at time $t$. The Hermiticity requirement implies that $\|A(t)\|=\|A(0)\|$ for all $t>0$, known as the Bessel equality. It means that the magnitude or the length of the vector is a constant of motion. This is a useful property, e.g., for the verification of the result. The space on which we are operating is not abstract but realized. Hence $d$ may be finite as we shall see in this problem.

Since there is always one degree of freedom in choosing one basis vector initially, we choose $f_{0}=A$. This choice yields boundary conditions on $a_{k}(t)$ 's:

$$
a_{k}(t=0)= \begin{cases}1 & \text { if } k=0 \\ 0 & \text { if } k=1,2, \ldots\end{cases}
$$

Observe also that the orthogonality property gives

$$
a_{0}(t)=\frac{(A(t), A)}{(A, A)}
$$

sometimes known as the relaxation function, the most basic time dependent function as we shall see.

Given Eqs. (1), (2) and (3), the recurrence relations method rests on the following fact: If the inner product means the Kubo scalar product, both $f_{k}$ and $a_{k}$ satisfy certain unique recurrence relations. They are threeterm recurrence relations except the basal ones $(k=0)$ which have only two terms. They are given below: For $k=0,1, \ldots, d-1$,

$$
\begin{gathered}
f_{k+1}=\dot{f}_{k}+\Delta_{k} f_{k}, \\
\Delta_{k+1} a_{k+1}(t)=-\dot{a}_{k}(t)+a_{k-1}(t),
\end{gathered}
$$

where $\Delta_{k}=\left\|f_{k}\right\| /\left\|f_{k-1}\right\|, f_{1}=a_{-1} \equiv 0, \Delta_{0} \equiv 1$. Here $\|f\|=(f, f)$. These ratios of successive norms are called recurrants. Notice that our definition of a norm is nonstandard but more convenient for our purposes. There will be no confusion by this usage. Equations (5) and (6) are referred to in the literature as the RR1 and RR2, respectively.

Observe that given $f_{0}$, the other or higher $f_{k}$ 's can be obtained quite systematically. The basis vectors are thus hierarchic, successively representing increasing dimensions of the realized space. The projection coefficients cannot enjoy this property since the basal one $a_{0}(t)$ is not known.

The recurrence relations methods means that Eqs. (5) and (6), or RR1 and 2, are to be solved. Their solutions are then the solutions for Eq. (1). This has been already demonstrated in numerous examples [5-15].

There is one particularly important relation, which follows from Eq. (6),

$$
\Delta_{1} a_{1}(t)=-\dot{a}_{0}(t)
$$

This is one of the basal relations, which follows from Eq. (6) by setting $k=0$. The right hand side (rhs) of Eq. (7) denotes the relaxation function (see Eq. (4)). The left-hand-side (lhs) of Eq. (7) denotes the response function or fluctuations, e.g., $\langle A(t) A>$. The connection between these two physical quantities is central to dynamic theory, known as the fluctuation dissipation theorem [17]. 
Let us define $\tilde{a}_{k}(z)=L a_{k}(t)$, where $L$ is the Laplace transform operator. If $L$ is applied to Eq. (6), we obtain

$\tilde{a}_{0}(z)=1 / z+\Delta_{1} / z+\Delta_{2} / z+\ldots+\Delta_{d-1} / z \equiv 1 / z+\Delta_{1} \tilde{b}_{1}(z)$,

a continued fraction of order $d-1$. The second term on the rhs of Eq. (8), a continued fraction of order $d-2$, is known as the memory function in the generalized Langevin equation [18]. There is a convolution relation between $a_{1}$ and $b_{1}$.

\section{Application to the Ising model}

Henceforth we shall adopt the notation $S_{i}^{x}=x_{i}, S_{i}^{y}=$ $y_{i}$, and $S_{i}^{z}=z_{i}$, which will simplify our presentation. We caution that the subscripts on $f_{k}(k=0,1,2, \ldots)$ are not to be confused with the subscripts on, e.g., $x_{j}$. There are no relations between the two.

In this section we will consider the Ising model in $1 D$ (linear chain) with periodic boundary conditions imposed. This $1 D$ model is found to contain the basic structure of the Ising dynamics. Hence we will show explicit details of our calculation. Afterward we will remark how the conclusion is generalized to higher $D$ 's.

\section{III.1 Recurrence relations analysis for 1D Ising model}

In terms of our new notation, for $1 D$

$$
\begin{gathered}
H=-J \sum_{i} S_{i}^{z} S_{i+1}^{z}=-J \sum_{i} z_{i} z_{i+1}, \\
f_{0}=A=S_{0}^{x}=x_{0} .
\end{gathered}
$$

Using the RR1 (Eq. (5)),

$$
f_{1}=J\left(y_{0} z_{1}+z_{-1} y_{0}\right) .
$$

Hence,

$$
\begin{gathered}
\left\|f_{1}\right\|=\frac{J^{2}}{2}\left[\left(x_{0}, x_{0}\right)+4\left(x_{0}, z_{-1} x_{0} z_{1}\right)\right], \\
\Delta_{1}=\frac{\left\|f_{1}\right\|}{\left\|f_{0}\right\|}=\frac{J^{2}}{2}(1+4 \Psi),
\end{gathered}
$$

where

$$
\Psi=\frac{\left(x_{0}, z_{-1} x_{0} z_{1}\right)}{\left(x_{0}, x_{0}\right)}
$$

Note that the $x x$ component of the susceptibility $\chi^{x x} \equiv$ $\chi=N\left(x_{0}, x_{0}\right)$, where $N$ is the total number of spins in the system. We can also calculate Eq. (13) by Kubo's theorem [1],

$$
\left\|f_{1}\right\|=\left(f_{1}, f_{1}\right)=i \beta^{-1}<\left[f_{1}, f_{0}\right]>=\frac{2 J}{\beta}<z_{0} z_{1}>,
$$

where

$$
<z_{0} z_{1}>=\frac{1}{4} \tanh K
$$

where $K=\beta J / 4$ and $\beta=1 / k_{B} T$ [19]. We shall later exploit the equality between Eqs.(12) and (15) to obtain an explicit form for $\Psi$, an important quantity for the dynamic analysis.

Given $\Delta_{1}$ by Eq. (13), we are now in the position to obtain $f_{2}$ by the RR 1 ,

$$
f_{2}=\dot{f}_{1}+\Delta_{1} f_{0}
$$

Using Eqs. (11) and (13) in Eq. (17), we obtain

$$
\begin{gathered}
f_{2}=2 J^{2}\left(\Psi x_{0}-z_{-1} x_{0} z_{1}\right), \\
\left\|f_{2}\right\|=\frac{J^{2}}{4}\left(1-16 \Psi^{2}\right),
\end{gathered}
$$

and

$$
\Delta_{2}=\frac{J^{2}}{2}\left(1-4 \Psi^{2}\right)
$$

Continuing this way we next look at $f_{3}$ by the RR1

$$
f_{3}=\dot{f}_{2}+\Delta_{2} f_{1} .
$$

Using Eqs. (11), (18), and (20) in Eq. (21) we find that

$$
f_{3}=0
$$

hence also $\Delta_{3}=0$..

Thus we have arrived at an essential result that the realized Hilbert space for $A(t)=x_{0}(t)$ has but three dimensions, spanned by $f_{0}, f_{1}$, and $f_{2}$ only. The shape of the space is determined by the two recurrants $\Delta_{1}$ and $\Delta_{2}$. The trajectory of this vector, which is constrained to the surface of this space, is closed.

Given the two recurrants, we can now obtain $a_{0}, a_{1}$ and $a_{2}$ by the RR2 (Eq. (6)) or also $\tilde{a}_{0}$ by Eq. (8) and then by inverse transform $L^{-1}$. They are

$$
\begin{gathered}
a_{0}(t)=\frac{1}{\omega^{2}}\left(\Delta_{2}+\Delta_{1} \cos \omega t\right), \\
a_{1}(t)=\frac{\sin \omega t}{\omega} \\
a_{2}(t)=\frac{1}{\omega^{2}}(1-\cos \omega t),
\end{gathered}
$$

where $\omega=J / \hbar$ (but $\hbar=1$ ), and $\Delta_{1}$ and $\Delta_{2}$ given up to the function $\Psi$ by Eqs. (13) and (20), respectively. Observe that Eqs. (23) to (25) satisfy the boundary conditions (see Eq. (3)). In addition, Eqs. (23) and (25) satisfy the basal RR2 (see Eq. (7)).

Finally we can write down the total time evolution

$$
x_{0}(t)=a_{0}(t) f_{0}+a_{1}(t) f_{1}+a_{2}(t) f_{2},
$$


where for each term on the rhs we have found an explicit expression. The validity of Eq. (26) can be further tested through the Bessel equality $\left\|x_{0}(t)\right\|=\left\|x_{0}\right\|$. Noting the orthogonality, we obtain

$$
\frac{\left\|x_{0}(t)\right\|}{\left\|x_{0}\right\|}=\left(a_{0}\right)^{2}+\left(a_{1}\right)^{2} \Delta_{1}+\left(a_{2}\right)^{2} \Delta_{1} \Delta_{2}=1,
$$

where the final result is obtained by substituting various identities already obtained above.

\section{III.2 Dynamical implications}

Using Eq. (26) we can obtain a number of dynamical results. For example, we can immediately determine the dynamic structure function $\left\langle x_{0}(t) x_{0}\right\rangle$ as follows: From Eq. (26)

$$
\begin{gathered}
\frac{S(t)}{N}=<x_{0}(t) x_{0}>=\frac{1}{4} a_{0}(t)-i J<z_{0} z_{1}>a_{1}(t)+ \\
\frac{J^{2}}{2}\left(\Psi-<z_{1} z_{2}>\right) a_{2}(t)
\end{gathered}
$$

where we have used $\left\langle f_{1} x_{0}>=-i J<z_{0} z_{1}>\right.$ and $<f_{2} x_{0}>=J^{2} / 2\left(\phi-<z_{0} z_{2}>\right)$. If we define

$$
\tilde{S}(z)=L S(t)=\tilde{R}(z)+i \tilde{I}(z),
$$

the real and imaginary parts $\tilde{R}$ and $\tilde{I}$ (which can be read off from Eq. (28)) are related through KramersKronig relations [1].

As a scattering problem, the term $\tilde{I}(z)$ would denote the absorptive part. Thus the dynamic susceptibility $\tilde{\chi}(z)$ is contained in $\tilde{I}(z)$ which we shall prove below: From the recurrence relations theory [10]

$$
\Delta_{1} \tilde{a}_{1}(z)=\frac{\tilde{\chi}(z)}{\chi}
$$

where $\chi=N\left(x_{0}, x_{0}\right)$. Now

$$
\Delta_{1}=\frac{J^{2}}{2}(1+4 \Psi)=\frac{2 J}{\beta} \frac{<z_{0} z_{1}>}{\left(x_{0}, x_{0}\right)},
$$

where the second equality is obtained by applying the Kubo theorem to $\left\|f_{1}\right\|$ (see Eq. (15)). Hence,

$$
2 J<z_{0} z_{1}>=\beta \Delta_{1}\left(x_{0}, x_{0}\right) .
$$

Observe that the lhs of the above is the static term conjugate to $a_{1}(t)$, the second term in the rhs of Eq. (28), thus together corresponding to $\chi(t)=L^{-1} \tilde{\chi}(z)$.

One can also obtain the dynamic susceptibility using Eq. (26) in the definition [1]: For $t>0$,

$\chi(t)=i<\left[x_{0}(t), x_{0}\right]>=i a_{1}(t)<\left[f_{1}, x_{0}\right]>=\beta\left\|f_{1}\right\| a_{1}(t)$,

where $\left\|f_{1}\right\|=(2 J / \beta)<z_{0} z_{1}>=(J / 2 \beta) \tanh K$. Hence,

$$
\tilde{\chi}(z=0)=\frac{\beta}{J}\left\|f_{1}\right\| .
$$

We shall see in Section IV that $\tilde{\chi}(z=0)<\chi_{T}$, where $\chi_{T}$ means the isothermal susceptibility.

\section{III.3 Higher dimensions [20]}

The time evolution of $x_{0}$ in higher dimensions may be obtained in a similar manner as for $1 D$. The essential aspect in $1 D$ is that the realized Hilbert space has $d=3$. As alluded in Section I, the Hilbert space dimensions turn out to be simply related to the coordination number. For the Ising model in $D$ lattice dimensions the Hilbert space dimensions are:

$$
d=q+1
$$

where $q$ is the coordination number [20]. Thus, for example, in the honeycomb lattice there is but one more basis vector than in the linear chain. The correlation functions that enter depend on the lattice dimensions $D$. Otherwise the dynamic structures are determined solely by $q$ alone.

If $q \rightarrow \infty$, the model is known as the spin van der Waals model [21]. At this limit the dynamical picture changes drastically. As $d \rightarrow \infty$, the time correlation functions are no longer periodic. It has already been found through a recurrence relations analysis that if $T>T_{c}$

$$
a_{k}(t)=\frac{t^{k}}{k !} e^{-c t^{2}},
$$

where $c>0$ is a constant.

\section{Statics from Dynamics}

To obtain dynamic properties one ordinarily needs static properties as for example Eq. (26). Thus to think that static properties can be deduced from dynamic properties would seem quite unusual if not likely. But if the Hilbert space dimensionality $d$ is finite as in the Ising model (see Section III), we can in fact obtain certain static properties from dynamic results as we will illustrate here.

Consider the $x x$ component of the static susceptibility $\chi$,

$$
\chi=\left(\sum x_{i}, \sum x_{j}\right)=N\left(x_{0}, x_{0}\right)
$$

(Note that $\chi=\beta^{-1} \chi_{T}$, where $\chi_{T}$ is the isothermal susceptibility.) The susceptibility has this form, different from the $z z$ component, since $\left[H, x_{0}\right] \neq 0$. The inner product appearing in Eq. (37) is a kind of temperature integral, i.e.,

$$
\left(x_{0}, x_{0}\right)=\beta^{-1} \int_{0}^{\beta}<x_{0}(\lambda) x_{0}>d \lambda
$$

where

$$
x_{0}(\lambda)=\exp (\lambda H) x_{0} \exp (-\lambda H) .
$$


Hence if we know the "temperature" evolution of $x_{0}$, the rhs of Eq. (38) may be evaluated. But we already know the time evolution of $x_{0}$ (see Eq. (26)). If $t \rightarrow-i \lambda$ therein,

$$
x_{0}(\lambda)=a_{0}(-i \lambda) f_{0}+a_{1}(-i \lambda) f_{1}+a_{2}(-i \lambda) f_{3},
$$

where $a_{0}, a_{1}$ and $a_{2}$ can be immediately obtained from Eqs. (23), (24) and (25). If Eq. (40) is substituted in Eq. (38), we obtain

$$
\beta\left(x_{0}, x_{0}\right)=\frac{1}{4} I_{0}+i<f_{1} x_{0}>I_{1}+<f_{2} x_{0}>I_{2},
$$

where $I_{0}, I_{1}$ and $I_{2}$ are "temperature" integrals, easily evaluated. Using $\left.\left\langle f_{1} x_{0}\right\rangle=-i J<z_{0} z_{1}\right\rangle$ and $<f_{2} x_{0}>=J^{2} / 2\left(\Psi-<z_{0} z_{2}>\right.$ ) (see Section III), we obtain

$$
\begin{gathered}
\beta\left(x_{0}, x_{0}\right)=\frac{1}{8}\left[\left(1+\frac{\sinh u}{u}+8 \frac{(1-\cosh u)}{u}<z_{0} z_{1}>\right.\right. \\
\left.-\left(1-\frac{\sinh u}{u}\right)<z_{0} z_{2}>\right],
\end{gathered}
$$

where $u=\beta J$. Observe that the rhs does not contain $\Psi$. Using the results $\left\langle z_{0} z_{1}\right\rangle=1 / 4 \tanh u / 4$, $<z_{0} z_{2}>=(1 / 4 \tanh u / 4)^{2}$, and after some rearrangements we obtain

$$
8\left(x_{0}, x_{0}\right)=\operatorname{sech}^{2} K+\frac{\tanh K}{K},
$$

where $K=u / 4$. We have recovered the known result $[22]$.

The above result may be used to obtain an expression for $\Psi$. From the definition, Eq. (13) or (32),

$$
1+4 \Psi=\frac{2 \tanh K / K}{\tanh K / K+\operatorname{sech}^{2} K} .
$$

Hence,

$$
\Psi=\left(x_{0}, z_{-1} x_{0} z_{1}\right)=\frac{1}{4} \frac{1-2 K \operatorname{csch} 2 K}{1+2 K \operatorname{csch} 2 K} .
$$

Finally comparing with the zero-frequency limit of the dynamic susceptibility $\tilde{\chi}(z=0)$ (see Eq. (34)), we note the inequality [23]

$$
\chi_{T}>\tilde{\chi}(z=0) .
$$

In higher dimensions one can obtain, e.g., the susceptibility. But since the static correlation functions are not known except in $2 D$, these new results may not be as interesting as in $1 D$. However they can yield, e.g., high temperature expansions much more simply than the standard method [20].

We ought to mention that the static susceptibility $\chi(K)$ (see Eq. (43)) satisfies the bounds due to Falk and Bruch [24],

$$
\tanh p / p \leq \frac{\chi(K)}{Y(K)} \leq 1
$$

where

$$
Y(K)=N<x_{2}^{2}>=N / 4
$$

and

$$
\left.p^{\prime} \tanh p^{\prime}\right|_{p}=2 K \tanh K .
$$

The lhs of Eq. (47) is known as a stronger lower bound. Our solution, Eq. (43), suggests that the stronger lower bound of Falk-Bruch may not be strong enough.

\section{Discussion}

That the time evolution of $x_{0}=S_{0}^{x}$ requires a finite number of the basis vectors is perhaps most remarkable. It implies dynamically that if energy is imparted to this spin by some external perturbation, it does not become delocalized. This energy goes back and forth between its neighbors, describing in effect a periodic motion. From the perspective of the Hilbert space, it delineates a closed trajectory. We must conclude therefore that the time evolution in this case is not ergodic in the sense of the usual meaning of this word.

If the interactions contained other terms (e.g., $X X$ interactions), the dynamics would change [25]. The energy would become delocalized as there are numerous other nonstationary spin states. The dimensions of the realized Hilbert space would become infinitely large and the trajectory would be no longer closed, but open. In certain static limits some exact solutions have been found by the recurrence relations method [5-16].

We have demonstrated in some detail for $1 D$ that the recurrence relations method is a powerful yet simple technique for obtaining very profound descriptions of dynamics for Hermitian systems. There are other properties such as the memory function, subspaces, not explored in this paper, which are of special significance to dynamical processes [26].

\section{References}

[1] W. Marshall and R.D. Lowde, Rep. Prog. Phys. 31, 705 (1968).

[2] M.H. Lee, Phys. Rev. B 26, 2547 (1982).

[3] M.H. Lee, Phys. Rev. Lett. 49, 1072 (1982).

[4] M.H. Lee, J. Math. Phys. 24, 2512 (1983).

[5] J. Florencio and F.C. Sá Barreto, Phys. Rev. B 60, 9555 (1999).

[6] R.S. Sinkovits and S. Sen, Phys. Rev. Lett. 74, 2686 (1995).

[7] I. Sawada, Phys. Rev. Lett. 83, 1668 (1999).

[8] S.G. Jo, K.H. Lee, S.C. Kim, and S.D. Choi, Phys. Rev. E 55, 3676 (1997).

[9] I.M. Kim and B.Y. Ha, Can. J. Phys. 67, 31 (1989). 
[10] J. Hong and M.H. Lee, Phys. Rev. Lett. 70, 1972 (1993)

[11] J. Kim and I. Sawada, Phys. Rev. E 61, R2172 (2000).

[12] V.S. Viswanath and G. Müller, Recursion Method (Springer-Verlag, Berlin, 1994).

[13] E.B. Brown, Phys. Rev. B 45, 10809 (1992); 49, 4305 (1994).

[14] D. Vitali and P. Grigolini, Phys. Rev. A 39, 1486 (1989).

[15] A.S.T. Pires, Helv. Phys. Acta 61, 988 (1988); Phys. St. Sol. b 129, 136 (1985).

[16] J.F. Annett et al., J. Phys. Cond. Matter 6, 6455 (1994).

[17] R. Kubo, Rep. Prog. Phys. 29, 235 (1966).
[18] U. Balucani, V. Tognetti, and R. Valluauri, Phys. Rev. A 19, 177 (1979).

[19] H.E. Stanley, Introduction to Phase Transitions and Critical Phenomena (Oxford Univ. Press, Oxford, 1971).

[20] S. Sen, Ph.D. Thesis, University of Georgia, 1990.

[21] M.H. Lee, I.M. Kim, and R. Dekeyser, Phys. Rev. Lett. 52, 1579 (1984).

[22] M.E. Fisher, J. Math. Phys. 4, 124 (1963).

[23] H. Falk, Phys. Rev. 165, 602 (1968).

[24] H. Falk and L.W. Bruch, Phys. Rev. 180, 442 (1969).

[25] J. Florencio and M.H. Lee, Phys. Rev. B 35, 1835 (1987).

[26] M.H. Lee, Phys. Rev. E 61, 3571 (2000). 